\title{
NUMERICAL INVESTIGATION OF ENGINE INLET VANE HOT-AIR ANTI-ICING SYSTEM WITH SURFACE AIR FILM
}

\author{
HUI MA, WEIJIAN CHEN AND DALIN ZHANG \\ College of Aerospace, Nanjing University of Aeronautics and Astronautics, No.29 Yudao Street \\ Nanjing, Jiangsu 210016, PR China \\ valen@nuaa.edu.cn
}

\begin{abstract}
The inlet vane of aircraft engine needs to be equipped with anti-icing system to prevent ice accretion on the leading edge due to flight safety requirements, and the engine bleed hot-air is mostly used to heat the vane surface in anti-icing system. In order to save the energy consumption, a new anti-icing structure was developed and investigated with numerical simulation. Besides the use of small tunnels to enhance the heat transfer characteristics, a narrow gap was opened and assigned on the vane surface at the end of the anti-icing tunnels, and the exhaust hot-air was released from the gap to form an air film on the outside surface, which was supposed to prevent the droplets from impinging to the surface and sweep the droplets away. The droplets impingement on the vane surface was investigated by solving the $3 \mathrm{D}$ Eulerian air/droplets twophase model, and the impingement results were compared with the original system. Meanwhile, the thermodynamic analysis of the anti-icing system was presented in this paper. The results indicate that the air film is effective to decrease the droplets impingement area, and the new structure could provide more heat flux for anti-icing than the regular anti-icing structure.
\end{abstract}

Keywords: Anti-icing; engine inlet vane; aircraft icing; numerical simulation.

\section{Introduction}

Ice usually accretes on the exposed frontal surfaces when aircraft flying under icing conditions, which causes severe threat to flight safety. Ice accretion might occur on both the inlet lip and vane of the engine, which will degrade the engine aerodynamic performance. And even more seriously, the shedding ice may cause the structural damage to the inner blades and other components. Anti-icing system must be equipped for the engine, and the hot-air anti-icing system which uses the bleed hot air from engine compressor is widely used to prevent the engine from icing. The bleed hot air could heat the surfaces to evaporate the impinging super-cold water droplets.

There have been a few investigations on the engine hot-air anti-icing system of inlet lip ${ }^{1-7}$ while few were reported on the inlet vane anti-icing system. Nowadays many investigations focus on the structure optimization to improve the efficiency and decrease the usage of the engine bleed hot air so that not to degrade the engine performance significantly ${ }^{1}$. The numerical simulation is an effective way to investigate the droplets impingement, the flow and heat transfer of hot air anti-icing system. 
Based on the analysis of a current-in-use system, a new engine inlet vane hot-air antiicing structure was introduced and numerically simulated in this paper. The small tunnels are used to enhance the convective heat transfer, and the exhaust hot air is released from a narrow gap at the end of the tunnels to form an air film to lessen the impinging region and heating area. Multi-block structured mesh is adopted in the numerical simulation to deal with the complicated structure, and the Eulerian air/droplets two-phase equations are solved to get the impinging characteristics. RANS (Renoylds Average Navier-Stokes) equations combined with SST turbulence model are solved to handle the coupled heat transfer between the external and internal flow of the vane anti-icing system. Comparisons of impingement characteristic and heat transfer of the two systems are presented.

\section{Mathematical Mode}

\subsection{Governing Equations}

The flow and heat transfer of the external and internal air of the hot-air anti-icing system was got by solving RANS equations combined with SST turbulence model. 3D Eulerian air/droplets two-phase model is solved for droplets impingement computation. The air phase equation of the droplets model is the classic RANS equations, so the governing equations for the air flow and heat transfer are given as following along with the air/droplets two-phase model.

According to the two-fluid model theory, the droplets phase is regarded as a pseudofluid interpenetrating with the gas phase, and the droplets governing equations are the same as single phase flow just to consider the interaction between two phases. The volume fraction of droplets $\alpha$ is defined as $\alpha=V_{w} / V$ where $V_{w}$ is the volume of droplets and $V$ is the control volume, so that the volume fraction of air phase is $\beta=1-\alpha$.

Under the icing conditions with low temperature and tiny droplets diameter, there are some assumptions as following ${ }^{8}$ :

(i) The droplets are uniformly distributed in the cloud and are spherical without any deformation, breaking, collision and coalescence or splashing;

(ii) No heat and mass transfer between the droplets and the surrounding air, which means the droplets will not evaporate, and the physical property parameters will be steady;

(iii) Turbulence effects on the droplets are neglected;

(iv) The only forces acting on the droplets are thus due to air drag, gravity and buoyancy.

Since the median volume diameter (MVD) is at the magnitude of $\mu \mathrm{m}, \alpha$ is of the order of about $10^{-6}$. The droplets volume can be ignored in the air phase equations, meaning $\beta \approx 1$.

The governing equations are presented here. 
Continuity equations:

air:

$$
\frac{\partial \rho}{\partial \tau}+\frac{\partial}{\partial x_{j}}\left(\rho u_{j}\right)=0
$$

droplets:

$$
\frac{\partial}{\partial \tau}\left(\alpha \rho_{w}\right)+\frac{\partial}{\partial x_{j}}\left(\alpha \rho_{w} u_{w j}\right)=0
$$

Momentum equations:

air: $\quad \frac{\partial}{\partial \tau}\left(\rho u_{i}\right)+\frac{\partial}{\partial x_{j}}\left(\rho u_{j} u_{i}\right)=-\frac{\partial p}{\partial x_{i}}+\frac{\partial}{\partial x_{j}}\left[\mu\left(\frac{\partial u_{j}}{\partial x_{i}}+\frac{\partial u_{i}}{\partial x_{j}}\right)\right]-\frac{\partial}{\partial x_{j}}\left(\rho \overline{u_{j}^{\prime} u_{i}^{\prime}}\right)$,

droplets: $\quad \frac{\partial}{\partial \tau}\left(\alpha \rho_{w} u_{w i}\right)+\frac{\partial}{\partial x_{j}}\left(\alpha \rho_{w} u_{w i} u_{w j}\right)=F_{d w i}+\alpha\left(\rho_{w}-\rho\right) g_{i}$.

Energy equation

air:

$$
\frac{\partial}{\partial \tau}(\bar{\rho} \hat{E})+\frac{\partial}{\partial x_{j}}\left(\bar{\rho} \hat{E} \tilde{u}_{j}+\tilde{u}_{j} \bar{p}\right)=\frac{\partial}{\partial x_{j}}\left(\tilde{u}_{i} \tilde{\tau}_{i j}-q_{j}\right)-S_{k}
$$

The energy equation for droplets is ignored according to the assumption. Where $\rho$ is density, $u$ is velocity, and $g$ is gravity. The suffix $\mathrm{w}$ represents variables related to droplets.

$F_{d w i}$ in Eq. (4) is the air drag terms to droplets,

$$
F_{d w i}=\frac{1}{2} \alpha \rho\left|u_{w i}-u_{i}\right|\left(u_{w i}-u_{i}\right) C_{D} \frac{\pi d^{2}}{4} / \frac{\pi d^{3}}{6},
$$

where $d$ is MVD, $C_{D}$ is drag coefficient.

Define the relative Reynolds number between air and droplets: $\operatorname{Re}_{w i}=\rho\left|u_{i}-u_{w i}\right| d / \mu, C_{D}$ has the following correlation laws at different $\operatorname{Re}_{w i}$ :

Newton:

$$
C_{D}=0.44
$$

$\operatorname{Re}_{w i}>1000$

Wallis-kliachko

$$
C_{D}=\left(1+\operatorname{Re}_{w i}^{2 / 3} / 6\right) 24 / \operatorname{Re}_{w i}
$$$$
1<\operatorname{Re}_{w i}<1000
$$

Stockes:

$$
C_{D}=24 / \mathrm{Re}_{w i}
$$$$
\operatorname{Re}_{w i}<1
$$

Substitute Eq. (6) into Eq. (4), the droplets momentum equation becomes:

$$
\frac{\partial}{\partial \tau}\left(\alpha \rho_{w} u_{w i}\right)+\frac{\partial}{\partial x_{j}}\left(\alpha \rho_{w} u_{w i} u_{w j}\right)=\frac{3}{4} \alpha \frac{C_{D} \operatorname{Re}_{w i}}{d^{2} / \mu}\left(u_{i}-u_{w i}\right)+\alpha\left(\rho_{w}-\rho\right) g_{i} .
$$

The above mathematical model of droplets is a dilute, weakly coupled air/droplets two-phase flow model. Noticing that the air phase equations are not coupled with the droplets equations, the air phase equations are just the classic RANS, and could be solved 
independently combined with SST two equations turbulence model. When the air flow field is acquired, droplets equations can be solved to get the droplets velocity and the impingement on the vane surface.

For the convenience of following analysis, the nominal density is defined as $\alpha \cdot \rho_{w}$, which represents the mass of water in unit volume.

\section{Numerical Method}

\subsection{Air flow field and heat transfer computation}

The governing equations are discretized with FVM method on multi-block structured mesh, and the convective terms are discretized with 3rd SMRAT format. The SIMPLE algorithm extended to the compressible region is adopted to handle the coupling among the velocity, pressure and density. In the solid region of vane, the energy equation with only diffusion terms is solved. The SIP method is used to handle the algebraic equations after discretization.

The far field Mach and temperature are given for the external flow computation, and the supply inlet pressure and temperature of the internal hot air are also provided.

\subsection{Droplets computation}

When droplets sweep past the inlet vane surface, aft the impingement limit it would produce a shadow zone where there is no droplets in that region (Droplets volume fraction $\left.\alpha=0, \alpha \cdot \rho_{w}=0\right)$. The above set of droplets equations is hyperbolic and also must be solved in the whole flow field, so it needs a reasonable treatment to the shadow zone and proper boundary conditions imposed to the vane surface.

Since the droplets nominal density $\alpha \cdot \rho_{w}=0$ in the shadow zone, velocity in the shadow zone has no definition which also means any velocity would match those equations. Virtual droplets with reasonable velocity and zero nominal density (usually set it to a tiny magnitude in the computation) are assumed to exist in the shadow zone, so the governing equations could be solved in the whole flow region and the shadow zone would not transfer any information to other regions.

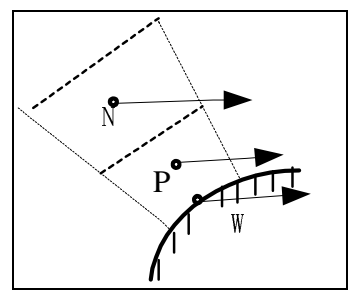

(a)

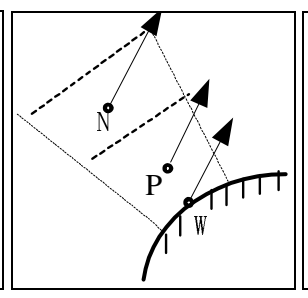

(b)

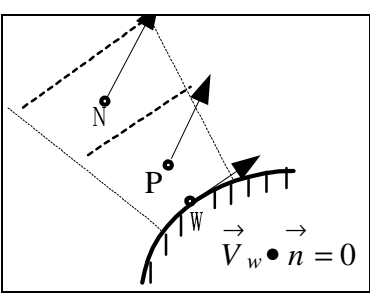

(c)

Fig. 1 Boundary condition of droplets impingement calculation 
For the wall boundary conditions, the vane surface is taken as an absorber which absorbs the impinging droplets without passing any information into the interior region. Fig. 1 shows the velocity treatment in two cells adjacent to the wall. The droplets velocity on the wall $\vec{V}_{w}$ is acquired with second order extrapolation. However, when the wall velocity vector points away from the wall, as Fig. 1(a) shows, the slip boundary condition $\vec{V}_{w} \bullet \vec{n}=0$ is used as Fig. 1(c) shows, where $\vec{n}$ represents the surface unit normal vector. This method is able to handle the impingement zone and shadow zone smoothly and automatically.

Because the droplets mass and momentum equations are weakly coupled and the shadow zone needs a special velocity treatment, the following Eq. (8) is first solved to get a initial velocity field, and then the whole set of equations is solved in a segregated way.

$$
\frac{\partial}{\partial \tau}\left(u_{w i}\right)+\frac{\partial}{\partial x_{j}}\left(u_{w j} u_{w i}\right)=\frac{3}{4 \rho_{w}} \frac{C_{D} \operatorname{Re}_{w i}}{d^{2} / \mu}\left(u_{i}-u_{w i}\right)+\left(1-\frac{\rho}{\rho_{w}}\right) g_{i} .
$$

The droplets equations are discretized with FVM on the same mesh system as the air flow computation. The first order fully implicit scheme is used for the time discretization, and the first order upwind scheme for the convection terms. The influence of high order scheme to droplet impingement calculation has been discussed in Ref. 8 .

\section{Results and Discussion}

\subsection{Geometric configuration}

The engine inlet vane is made of steel with a thickness about $3 \sim 5 \mathrm{~mm}$, the chord length is about $50 \mathrm{~mm}$. The original used system is shown in Fig. 2. The hot bleed air enters the internal anti-icing cavity through the array of small jet holes and impinges on the internal surface of vane leading edge to provide the anti-icing heat flux. The exhaust air after heat transfer flows out of the anti-icing cavity through the rectangular gaps to form an air film on the external vane surface.

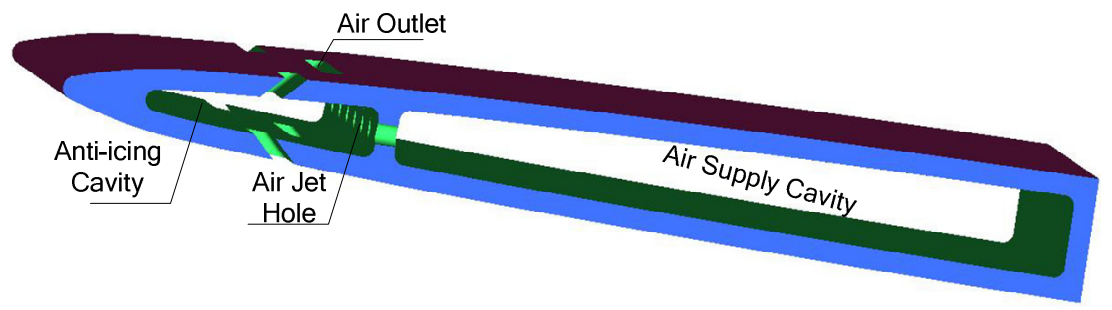

Fig. 2 Structure of original anti-icing system

The leading edge is the most concerned zone of anti-icing system because it usually catches most of the droplets. However, in the original system, the distance between jet holes and the leading edge internal surface is too large, and the diameter of jet holes is at 
the same magnitude as the height of anti-icing cavity, so it might not produce the impact jet flow effect on the surface which is originally supposed to improve the heat transfer coefficient on the internal surface. Meanwhile, small curvature of the inlet vane may lead to a large chord-wise impingement zone, the heating area needs to be extended in the following optimized design.

As Fig. 3 shows, the optimized system has small tunnels beneath the vane skin with the crossing size of $1 \mathrm{~mm} \times 0.5 \mathrm{~mm}$ to enhance the convective heat transfer, and the exhaust air of the anti-icing tunnels is released from the outlet gap to form an air film which is supposed to sweep away the impinging droplets. The position of outlet gap is moved back to the vane tail to cover the large impingement zone.

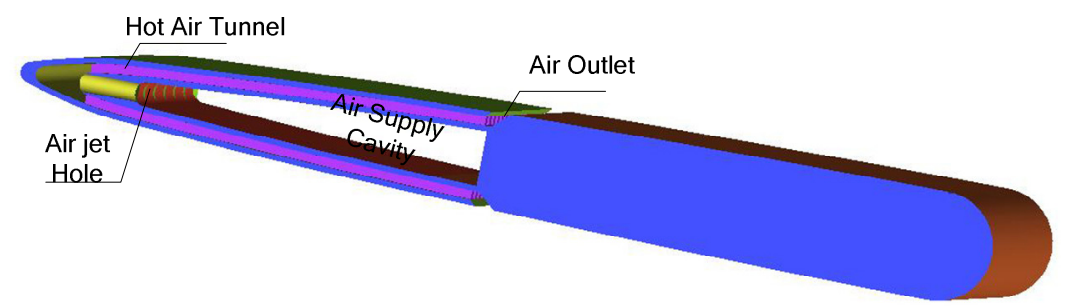

Fig. 3 Structure of optimized anti-icing system

\subsection{Computational mesh}

Due to the complicated geometric configurations, the multi-block structured mesh as Fig. 4 is adopted for the computation of the coupled heat transfer between the external and internal flow of the hot-air anti-icing system.

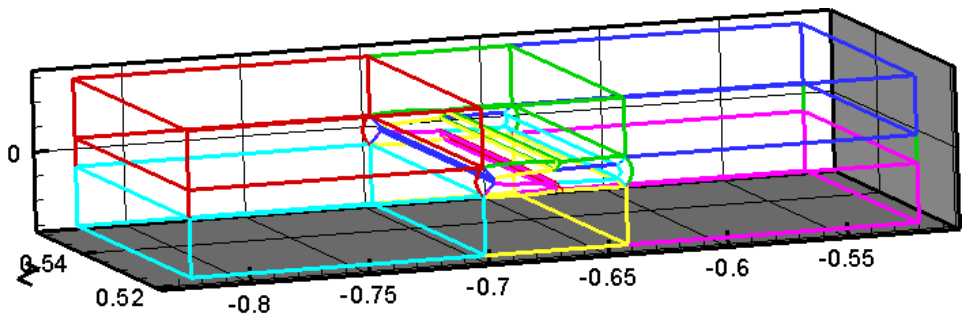

Fig. 4 Patched meshes

The internal flow computation is not necessary for the droplets computation and only the velocity at the hot air outlet is kept as the boundary condition, so the magnitude of computational nodes for the heat transfer analysis and droplets computation is at different level. About 5 millions of nodes are used for the coupled flow and heat transfer computation, and about 3 millions for the droplets computation. The mesh is refined in the near wall and the outlet zone. Fig. 5 shows the mesh around the vane, and Fig. 6 shows the cross section mesh of two structures. 


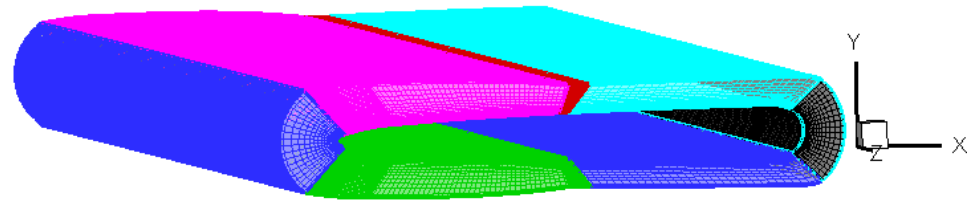

Fig. 5 Mesh around the vane
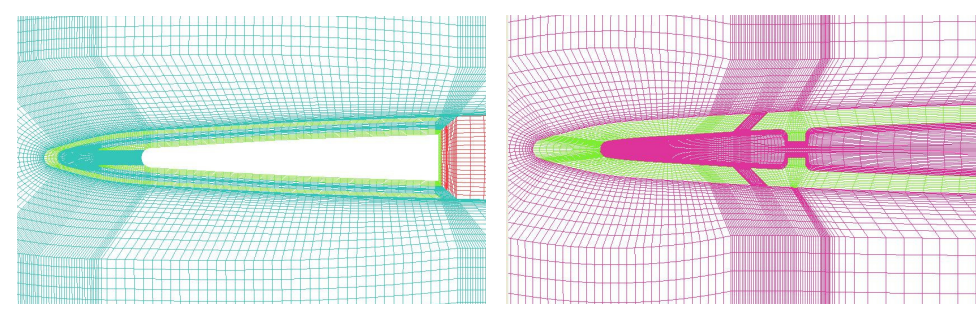

Fig. 6 Cross section mesh of the original and optimized system

\subsection{Results}

Impingement characteristic and convective heat transfer of the original and optimized structures are numerically simulated under the following given conditions: Supply hot-air temperature: $180^{\circ} \mathrm{C}$, supply pressure: $3 \times 10^{5} \mathrm{~Pa}$, far field mach number: 0.6 , far field temperature: $-5^{\circ} \mathrm{C}$, MVD: $20 \mu \mathrm{m}$, LWC: $1 \mathrm{~g} / \mathrm{m}^{3}$.

Fig. 7 shows the traces of droplets at the middle section of the original structure. The blue line is the multi-block patch line. There exists a large impinging region because the vane is in a sharp geometric shape. The droplets nominal density (Fig. 8) distribution indicates that the droplets are swept away from the vane surface near the hot-air outlet gaps (blue region) while impinge again on the surface just behind the gaps. Fig. 9 shows the nominal density distribution on the entire surface, and the blue region means no droplets impingement. The air film from the staggered array of outlet gaps does sweep away some of the droplets while a large impingement region still exists behind those gaps, so the air film has little influence on impingement and the original heating area is not enough for anti-icing.

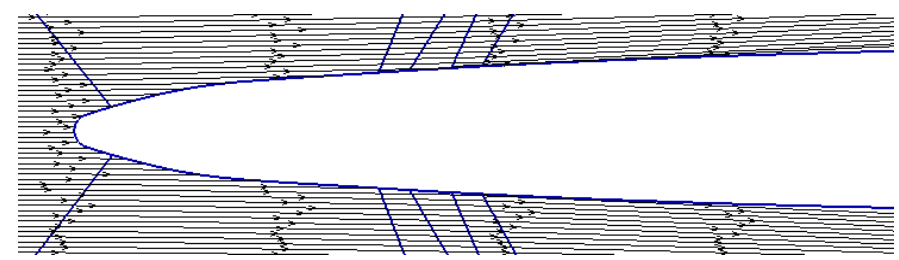

Fig. 7 Super-cooled droplets traces at the middle section (original system) 


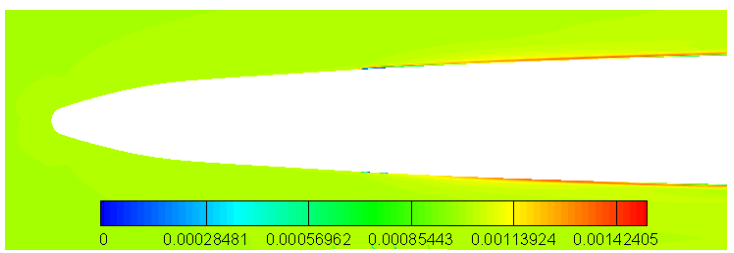

Fig. 8 Contour of droplets nominal density of the original system at the middle section $\left(\mathrm{kg} / \mathrm{m}^{3}\right)$

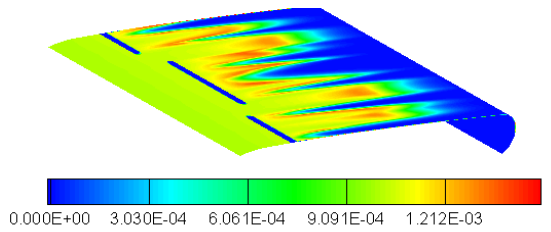

Fig. 9 Contour of droplets nominal density on surface of original system $\left(\mathrm{kg} / \mathrm{m}^{3}\right)$

Figs. 10 12 show the droplets impingement of the optimized system, and there is no droplets in the blue region. The nominal density distribution on the entire surface in Fig. 12 provides an obvious proof that the air film of optimized system prevents the droplets from impinging on the vane surface and reduces the impingement area effectively.

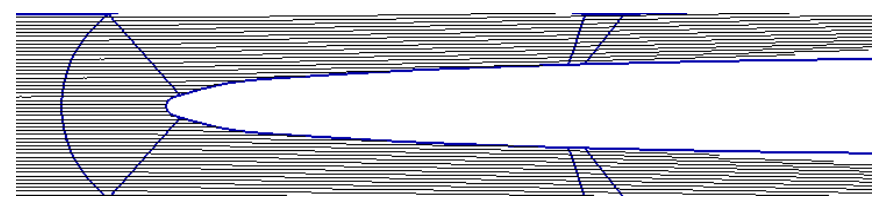

Fig. 10 Traces of super-cooled droplets at the middle section (optimized system)

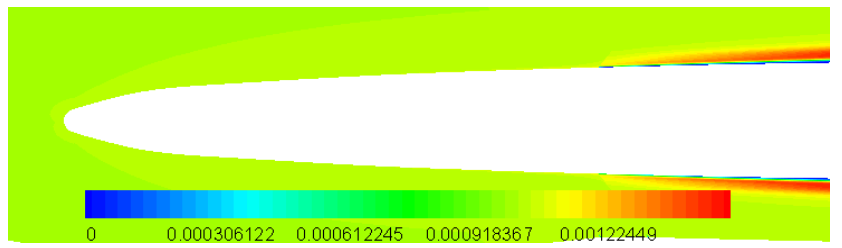

Fig. 11 Contour of droplets nominal density of optimized system at the middle section $\left(\mathrm{kg} / \mathrm{m}^{3}\right)$

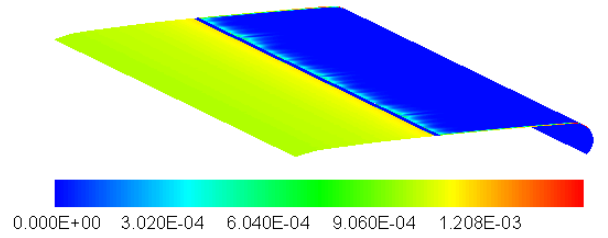

Fig. 12 Contour of droplets nominal density on surface of optimized system $\left(\mathrm{kg} / \mathrm{m}^{3}\right)$ 

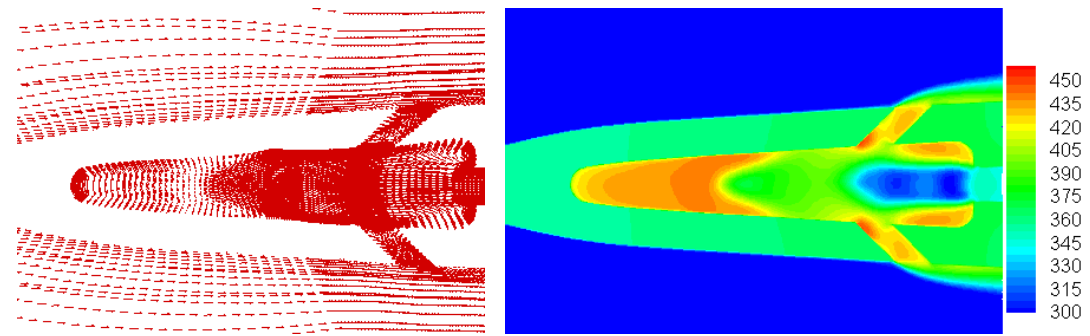

Fig. 13 Air velocity and temperature Contour of original system (K)
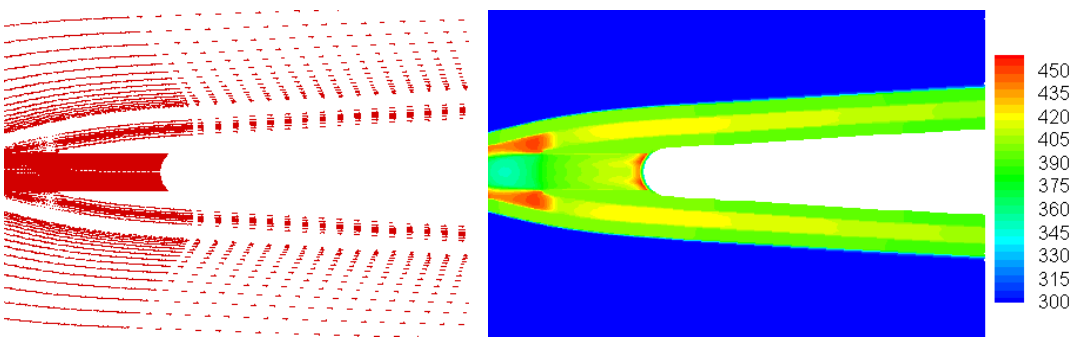

Fig. 14 Air velocity and temperature Contour of optimized system (K)

Fig. 13 and Fig. 14 show the air velocity vector and temperature field in the middle section at the leading edge of the two systems. In the original system (Fig.13), the impact jet flow effect is small due to the large distance between jet holes and leading edge. The exhaust hot-air flows out of the anti-icing cavity without sufficient heat transfer. It could not provide adequate heating to the leading edge where the largest droplet collection efficiency occurs and needs the most heat flux. On the other hand, as Fig. 14 shows, the improved structure could produce a rather better impact jet flow effect and the small hotair tunnel increases the convective heat transfer coefficient which could be seen from the temperature field.

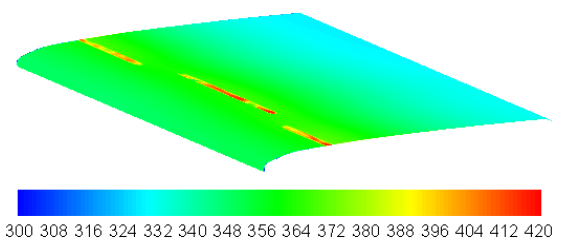

(a) original system

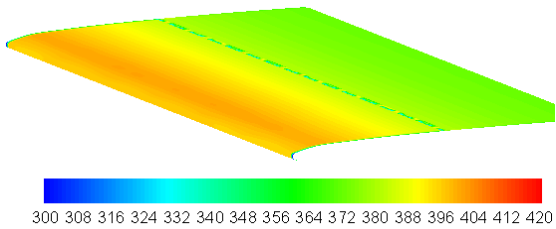

(b) optimized system

Fig. 15 Comparison of the external surface temperature of vane (K).

Fig. 15 shows the external surface temperature distribution of the vane. Under the same inlet pressure and temperature conditions, the optimized system has a higher surface temperature, and it could provide more anti-icing heat flux.

The above comparisons between the two systems indicate that small tunnels beneath the vane skin of the optimized system improve the convective heat transfer coefficient, 
and the surface air film formed by the exhaust hot-air reduces the droplets impinging area effectively. The optimized system has a better anti-icing performance and can also save hot-air consumption which helps to decrease engine bleeding loss.

\section{Conclusions}

A new structure of engine inlet vane hot-air anti-icing system with surface air film was numerically simulated and compared with the original configurations. Eulerian air/droplets two-phase equations are solved on multi-block structured meshes to compute 3-D droplets impingement. The coupled heat transfer computation of the external and internal flows was also investigated. The results show that the small tunnels improve the convection heat transfer coefficient, and could provide more heat flux to the leading edge. Also the surface air film decreases the impinging area efficiently. The optimized structure could meet the anti-icing requirement with less hot-air and reduces the adverse effect of the hot-air anti-icing system to engine performance.

\section{Acknowledgements}

This research work is supported by "the Fundamental Research Funds for the Central Universities”, NUAA Research Funding, NO. NS2010002.

\section{References}

1. M. Pellissier and W. Habish etc, AIAA, 2010-1238.

2. D. L. Rigby, AIAA, 2006-1012.

3. M. Fregeau, F. Saeed and I. Paraschivoiu, J. Air. 42, 665 (2005).

4. F. Saeed, M. Francois and I. Paraschivoiu, AIAA, 2000-630

5. F. Morency, M. T. Brahimi, F. Tezok and I. Paraschivoiu, AIAA, 98-0192.

6. J. S. Lu, Performance Investigation of Engine Inlet Hot Air Anti-icing System, MD Dissertation, Nanjing University of Aeronautics and Astronautics, 2008.

7. S. N. Chang and F. H. Han, J. BUAA, 25, 201 (1999).

8. W. J. Chen, Numerical Simulation of Ice Accretion on Airfoil, PHD Dissertation, Nanjing University of Aeronautics and Astronautics, 2007. 